\title{
Enantioselective orientation of chiral molecules induced by terahertz pulses with twisted polarization
}

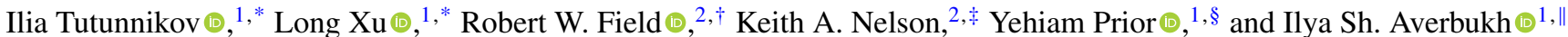 \\ ${ }^{1}$ AMOS and Department of Chemical and Biological Physics, The Weizmann Institute of Science, Rehovot 7610001, Israel \\ ${ }^{2}$ Department of Chemistry, Massachusetts Institute of Technology, Cambridge, Massachusetts 02139, USA
}

(Received 16 December 2020; accepted 24 February 2021; published 17 March 2021)

\begin{abstract}
Chirality and chiral molecules are key elements in modern chemical and biochemical industries. Individual addressing and the eventual separation of chiral enantiomers have been and still are important elusive tasks in molecular physics and chemistry, and a variety of methods have been introduced over the years to achieve these goals. Here, we theoretically demonstrate that a pair of cross-polarized $\mathrm{THz}$ pulses interacting with chiral molecules through their permanent dipole moments induces in these molecules an enantioselective orientation. This orientation persists for a long time, exceeding the duration of the THz pulses by several orders of magnitude, and its dependency on temperature and pulses' parameters is investigated. This persistent orientation may enhance the deflection of the molecules in inhomogeneous electromagnetic fields, potentially leading to viable separation techniques.
\end{abstract}

DOI: 10.1103/PhysRevResearch.3.013249

\section{INTRODUCTION}

Molecular chirality was discovered in the 19th century by Louis Pasteur [1] (for a historical excursion, see, e.g., Ref. [2]), and since then chirality has attracted considerable interest owing to its importance in physics, chemistry, biology, and medicine. Chiral molecules exist in two forms, called left- and right-handed enantiomers, which are mirror images of each other that cannot be superimposed by translations or rotations [3]. Even though the two enantiomers have many identical chemical and physical properties, e.g., boiling points and moments of inertia, they often differ in their biological activities. This is only one of the reasons why the abilities to differentiate, selectively manipulate, and separate the enantiomers in mixtures containing both of them are of great practical importance. From the point of view of fundamental physics, chiral molecules seem to be promising candidates for experiments aimed at measuring parity violation effects [4].

Some of the physical properties of the two enantiomers mimic their reflection relation. For example, the product of three Cartesian components of the molecular dipole moment has the opposite sign for the two enantiomers [5,6]. Utilizing the distinct properties of the enantiomers, a variety of

\footnotetext{
*These authors contributed equally to this work.

†rwfield@mit.edu

*kanelson@mit.edu

§yehiam.prior@weizmann.ac.il

"ilya.averbukh@weizmann.ac.il
}

Published by the American Physical Society under the terms of the Creative Commons Attribution 4.0 International license. Further distribution of this work must maintain attribution to the author(s) and the published article's title, journal citation, and DOI. methods for chiral discrimination using electromagnetic fields have been developed over the years, including microwave three-wave mixing spectroscopy [6-11], photoelectron circular dichroism [12-16], enantiospecific interaction with achiral magnetic substrates [17], Coulomb explosion imaging [18-20], and high-order harmonic generation [21]. Furthermore, various methods for enantiomeric interconversion have been proposed $[22,23]$. Using three orthogonally polarized resonant, phase-controlled electric fields, a state-specific enantiomeric enrichment has been demonstrated [24]. Most recently, a generic purely optical method for enantioselective orientation has been proposed [25-28] and experimentally demonstrated on propylene oxide molecule spun by an optical centrifuge [29] in which the polarization vector rotates unidirectionally with increasing angular velocity. This last method utilizes laser fields with "twisted" polarization [see Fig. 1(a)] and relies on the off-diagonal elements of the polarizability tensor which, in chiral molecules, have opposite signs for the two enantiomers. In related works, it was demonstrated that the optically induced orientation persists long after the end of the laser pulses [28,30]. For the purpose of the current work, fields with twisted polarization are understood to include pairs of delayed cross-polarized pulses [31-33] [see Fig. 1(b)], chiral pulse trains [34,35], polarization-shaped pulses [36-40], and the aforementioned optical centrifuge for molecules [41-45].

In this paper, we study the orientation of chiral molecules induced by terahertz $(\mathrm{THz})$ pulses with twisted polarization. With recent advances of $\mathrm{THz}$ pulse technology, intense $\mathrm{THz}$ pulses have been exploited for producing transient field-free orientation of polar molecules of various complexity [46-52]. In such experiments, orientation revival spikes periodically appear on long timescales, but the orientation signature generally rides on a zero baseline and its time average is exactly zero. Recently, it was shown that a single linearly polarized 


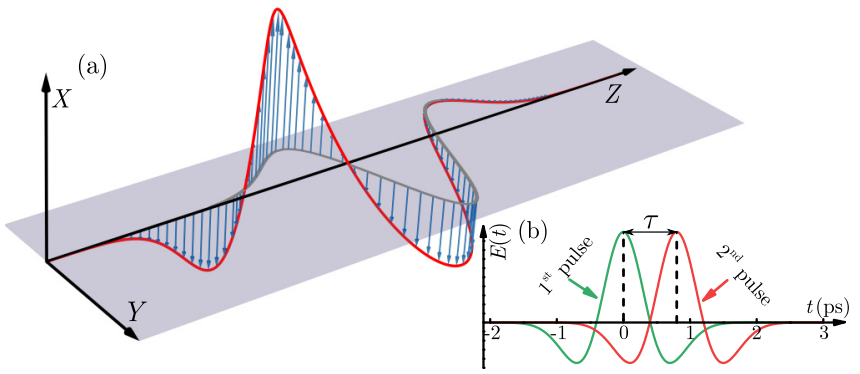

FIG. 1. (a) Illustration of the electric field with twisted polarization composed of two delayed cross-polarized $\mathrm{THz}$ pulses [see Eq. (5)]. The vertical blue arrows represent the $X$ projection of the field. The gray line in the $Y Z$ plane represents the $Y$ projection of the field. (b) Amplitude of the $X$-polarized (green) and the $Y$-polarized (red) pulses. Time dependence of the first pulse is given by $f(t)=$ $\left(1-2 a t^{2}\right) e^{-a t^{2}}$, while that of the second pulse by $f(t-\tau)$. Here, $a=3.06 \mathrm{ps}^{-2}$ and $\tau=0.80 \mathrm{ps}$. Time integral of the electric field is identically zero.

$\mathrm{THz}$ pulse induces persistent orientation in symmetric- and asymmetric-top molecules, including chiral molecules [53]. In contrast to the transient signal, persistent orientation means that the time-averaged postpulse orientation degree differs from zero on a long timescale, exceeding the duration of the $\mathrm{THz}$ pulse by several orders of magnitude.

Here, we theoretically demonstrate that when applied to chiral molecules, a pair of time-delayed cross-polarized $\mathrm{THz}$ pulses induces orientation in a direction perpendicular to the plane spanned by the input pulses' polarizations. This orientation is enantioselective namely, the two enantiomers are oriented in opposite directions relative to the plane. We show that the time-averaged projections of the molecular dipole moment onto all three laboratory axes remain nonzero on a nanosecond timescale.

\section{NUMERICAL METHODS}

We carried out classical as well as fully quantum mechanical simulations of the rotational dynamics of chiral molecules driven by a $\mathrm{THz}$ field. The Hamiltonian describing the molecular rotation driven by a $\mathrm{THz}$ field interacting with the molecular dipole moment is given by $\hat{H}(t)=\hat{H}_{r}+\hat{H}_{\text {int }}(t)$ $[54,55]$, where $\hat{H}_{r}$ is the rotational kinetic energy Hamiltonian and $\hat{H}_{\text {int }}(t)=-\hat{\boldsymbol{\mu}} \cdot \mathbf{E}(t)$ is the molecule-field interaction. Here $\hat{\boldsymbol{\mu}}$ is the molecular dipole moment operator and $\mathbf{E}(t)$ is the external electric field. In this work, the contributions of higher order interaction terms are small and are not included. For the quantum mechanical treatment, it is convenient to express the Hamiltonian in the basis of field-free symmetrictop wave functions $|J K M\rangle$ [56]. Here $J$ is the value of the total angular momentum (in units of $\hbar$ ), while $K$ and $M$ are the values of the projections on the molecule-fixed axis (here the axis with smallest moment of inertia) and the laboratoryfixed $Z$ axis, respectively. The nonzero matrix elements of the asymmetric-top kinetic energy operator are given by [56]

$$
\begin{array}{r}
\left\langle J K M\left|\hat{H}_{r}\right| J K M\right\rangle=\frac{B+C}{2}\left[J(J+1)-K^{2}\right]+A K^{2}, \\
\left\langle J K M\left|\hat{H}_{r}\right| J, K \pm 2, M\right\rangle=\frac{B-C}{4} f(J, K \pm 1),
\end{array}
$$

where $\quad f(J, K)=\sqrt{\left(J^{2}-K^{2}\right)\left[(J+1)^{2}-K^{2}\right]} ; \quad A=$ $\hbar^{2} / 2 I_{a}, B=\hbar^{2} / 2 I_{b}, C=\hbar^{2} / 2 I_{c}$ are the rotational constants $(A>B>C)$; and $I_{a}<I_{b}<I_{c}$ are the molecular moments of inertia. The time-dependent Schrödinger equation $i \hbar \partial_{t}|\Psi(t)\rangle=\hat{H}(t)|\Psi(t)\rangle$ is solved by numerical exponentiation of the Hamiltonian matrix (see Expokit [57]), and a detailed description of our numerical scheme can be found in Ref. [53]. In our simulations, the computational basis included all the rotational states with $J \leqslant 44$. For the case of propylene oxide molecules, which is used as an example, at a temperature of $T=5 \mathrm{~K}$, initial states with $J \leqslant 8$ are included in the thermal averaging.

In the classical limit, chiral molecules are modeled as asymmetric tops driven by an external torque. The classical equations of motion for the angular velocities (written in the frame of principal axes of inertia tensor) are the Euler's equations [58]

$$
\mathbf{I} \dot{\boldsymbol{\Omega}}=(\mathbf{I} \boldsymbol{\Omega}) \times \boldsymbol{\Omega}+\mathbf{T},
$$

where $\boldsymbol{\Omega}=\left(\Omega_{a}, \Omega_{b}, \Omega_{c}\right)$ is the angular velocity vector, $\mathbf{I}=$ $\operatorname{diag}\left(I_{a}, I_{b}, I_{c}\right)$ is the moment of inertia tensor. Here, the external torque vector $\mathbf{T}=\left(T_{a}, T_{b}, T_{c}\right)$ is given by $\mathbf{T}=\boldsymbol{\mu} \times Q \mathbf{E}$, where $\boldsymbol{\mu}$ is the molecular dipole moment vector and $Q$ is a $3 \times 3$ matrix composed of the quaternion's elements $[59,60]$. Quaternions are defined as quadruples of real numbers, $q=$ $\left(q_{0}, q_{1}, q_{2}, q_{3}\right)$, and obey the following equation of motion,

$$
\dot{q}=\frac{1}{2} q \Omega,
$$

where $\Omega=(0, \Omega)$ is a pure quaternion and the quaternion multiplication rule is implied [59,60]. To simulate the behavior of a classical ensemble, we use the Monte Carlo approach. For each individual asymmetric top, we numerically solve the system of Eqs. (3) and (4). We use ensembles consisting of $N=10^{7}$ molecules, which are initially isotropically distributed in space, and the initial angular velocities are given by the Boltzmann distribution $P\left(\Omega_{i}\right) \propto \exp \left(-I_{i} \Omega_{i}^{2} / 2 k_{B} T\right)$, $i=a, b, c$, where $T$ is the temperature and $k_{B}$ is the Boltzmann constant. The initial uniform random quaternions were generated using the recipe described in Ref. [61].

\section{SIMULATION RESULTS}

We consider propylene oxide (PPO) as a typical example of a chiral molecule. Table I summarizes the molecular properties of the right-handed enantiomer, $(R)$-PPO. Molecular moments of inertia and the components of molecular

TABLE I. Molecular properties of $(R)$-PPO: eigenvalues of the moment of inertia tensor (in atomic units) and components of dipole moment (in Debye) in the frame of molecular principal axes of inertia.

\begin{tabular}{lcc}
\hline \hline Molecule & $\begin{array}{c}\text { Moments } \\
\text { of inertia }\end{array}$ & $\begin{array}{c}\text { Molecular } \\
\text { dipole components }\end{array}$ \\
\hline$(R)$-propylene oxide & $I_{a}=180386$ & $\mu_{a}=0.965$ \\
& $I_{b}=493185$ & $\mu_{b}=-1.733$ \\
& $I_{c}=553513$ & $\mu_{c}=0.489$ \\
\hline \hline
\end{tabular}



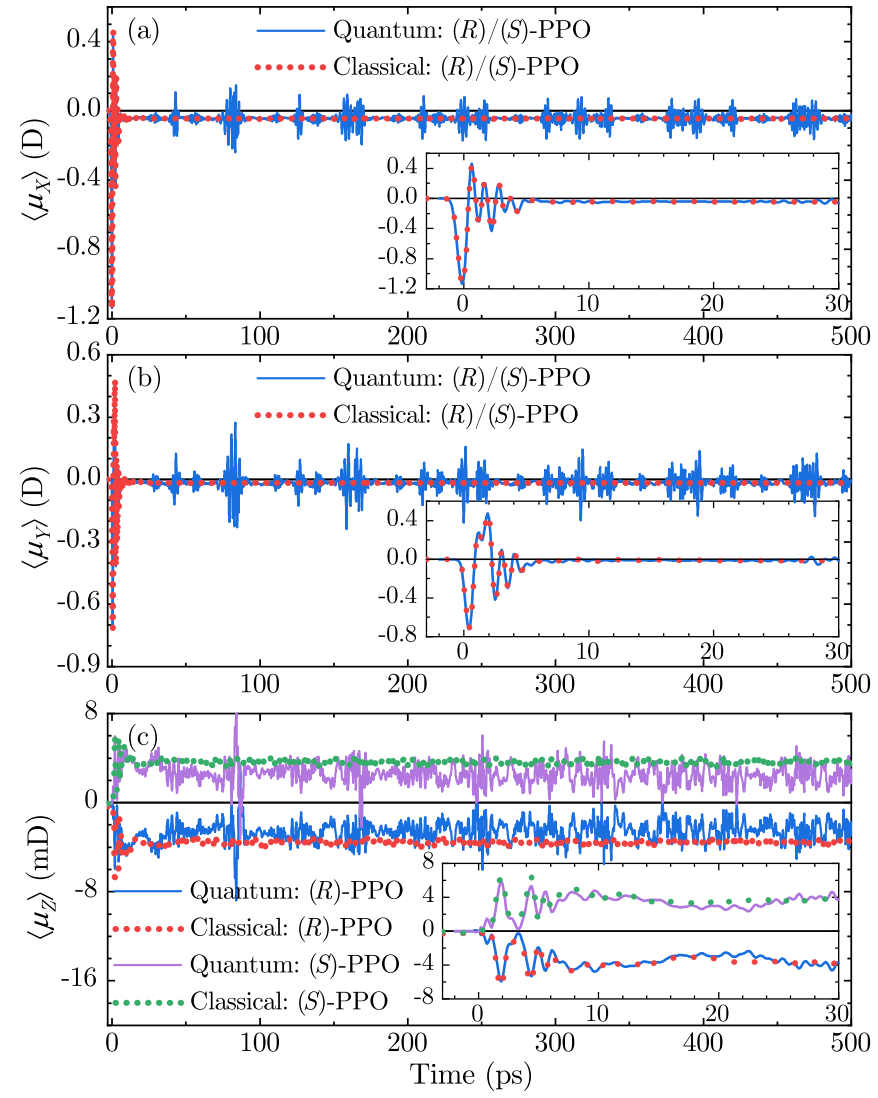

FIG. 2. Projections of the molecular dipole moment on the laboratory-fixed axes. Here $T=5 \mathrm{~K}$, and the $\mathrm{THz}$ field parameters are similar to Fig. 1, with $E_{0}=8.0 \mathrm{MV} / \mathrm{cm}$. The magnified insets show the first $30 \mathrm{ps}$ following the $\mathrm{THz}$ excitation, during which the overlap between the quantum and classical results is excellent. Note that in panel (c), the signals $\left\langle\mu_{Z}\right\rangle(t)$ are $\pi$ out of phase for the two enantiomers.

dipole moments were computed with the help of the GAUSSIAN software package (CAM-B3LYP/aug-cc-pVTZ method) [62]. The molecules are excited by a pair of delayed cross-polarized $\mathrm{THz}$ pulses. The combined electric field of the pulses is modeled using [50]

$$
\mathbf{E}(t)=E_{0}\left[f(t) \mathbf{e}_{X}+f(t-\tau) \mathbf{e}_{Y}\right],
$$

where $E_{0}$ is the peak amplitude, $f(t)=\left(1-2 a t^{2}\right) e^{-a t^{2}}$ defines the time dependence of each pulse, $a$ determines the temporal width of the pulse, $\tau$ is the time delay between the maxima of the two pulses, and $\mathbf{e}_{X}$ and $\mathbf{e}_{Y}$ are the unit vectors along the laboratory $X$ and $Y$ axes, respectively. The pulses propagate along the laboratory $Z$ axis, while $\mathbf{E}$ twists in the $X Y$ plane, as shown in Fig. 1.

Figure 2 shows the dipole moment projections along the three laboratory axes, $\left\langle\mu_{X}\right\rangle,\left\langle\mu_{Y}\right\rangle$, and $\left\langle\mu_{Z}\right\rangle$, as functions of time. The angle brackets $\langle\cdot\rangle$ denote the incoherent average of initial thermally populated rotational states, or the ensemble average in the classical case. The parameters of the field are $a=3.06 \mathrm{ps}^{-2}, \tau=0.80 \mathrm{ps}$, and $E_{0}=8.0 \mathrm{MV} / \mathrm{cm}$ (corresponding to the peak intensity of $8.5 \times 10^{10} \mathrm{~W} / \mathrm{cm}^{2}$ ); see Eq. (5) and Fig. 1. Note that THz pulses with peak amplitudes of tens of $\mathrm{MV} / \mathrm{cm}$, especially with the use of field enhance-

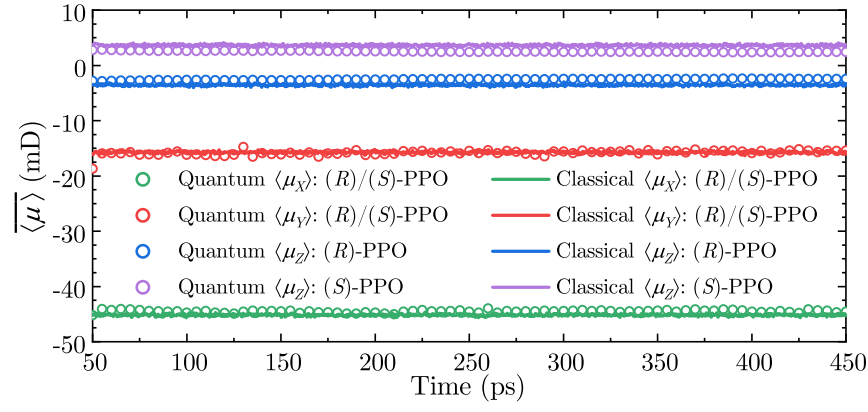

FIG. 3. Long-term behavior of the dipole projections shown in Fig. 2. Open circles represent sliding time average of the dipole signals $\left\langle\mu_{X}\right\rangle$ (green), $\left\langle\mu_{Y}\right\rangle$ (red), and $\left\langle\mu_{Z}\right\rangle$ (blue and purple) calculated quantum mechanically. The sliding time average is evaluated according to $\overline{\left\langle\mu_{i}\right\rangle(t)}=(\Delta t)^{-1} \int_{t-\Delta t / 2}^{t+\Delta t / 2} d t^{\prime}\left\langle\mu_{i}\right\rangle\left(t^{\prime}\right)$, where $\Delta t=100$ ps. For comparison, the correspondingly colored solid lines are the classical results.

ment structures [63-65], are experimentally available. As is evident from the insets in Fig. 2, on the short timescale the classical and quantum results are in excellent agreement. Each of the $\mathrm{THz}$ pulses is followed by a spike of dipole signal in the direction of the pulse polarization, i.e., initially along the $X$ axis and then along the $Y$ axis [see the minima in the insets of Figs. 2(a) and 2(b), which are before and after $t=0$, respectively]. This transient orientation induced by single $\mathrm{THz}$ pulses is expected and has been observed previously [46-53].

However, after the second pulse [see Fig. 2(c)], an unexpected result emerges: a dipole projection along the $Z$ axis (perpendicular to polarizations of both the pulses) appears. This orientation, which is unique to chiral molecules, is enantioselective, in the sense that the projections of the two enantiomers are of opposite signs, positive for $(S)$-PPO (the left-handed enantiomer) and negative for $(R)$-PPO. The enantioselectivity of the orientation in the $Z$ direction can be formally derived; for details, see Appendix A. A similar enantioselective orientation had been observed in chiral molecules which were optically excited by laser fields with twisted polarization acting on the molecular polarizability [26,27,29].

The classical results clearly show another remarkable feature of the induced orientation. After the field is switched off $[t>2.5 \mathrm{ps}$, see Fig. 1(b)], the dipole projections along all three laboratory axes persist on the nanosecond timescale. As can be seen from Fig. 3, on a timescale of up to $0.5 \mathrm{~ns}$, the time-averaged quantum signals reproduce well the steadystate dipole signals obtained by the classical calculation. On longer timescale, however, the direct quantum simulation deviates from the classical one, exhibiting quantum beats/revivals [66-68]. Classically, the persistent long-term orientation shown in Figs. 2 and 3 is in fact permanent.

In the absence of external torques (external fields), in the laboratory frame the angular momentum vector is conserved, while in the molecular frame the angular momentum follows a fixed trajectory, which can be visualized using the Binet construction [58]. Although the absolute orientation of an asymmetric top in the laboratory frame never recurs, depending on the energy and magnitude of the angular momentum, the projections of the molecular principal axes $a$ or $c$ on the conserved angular momentum vector have a constant 

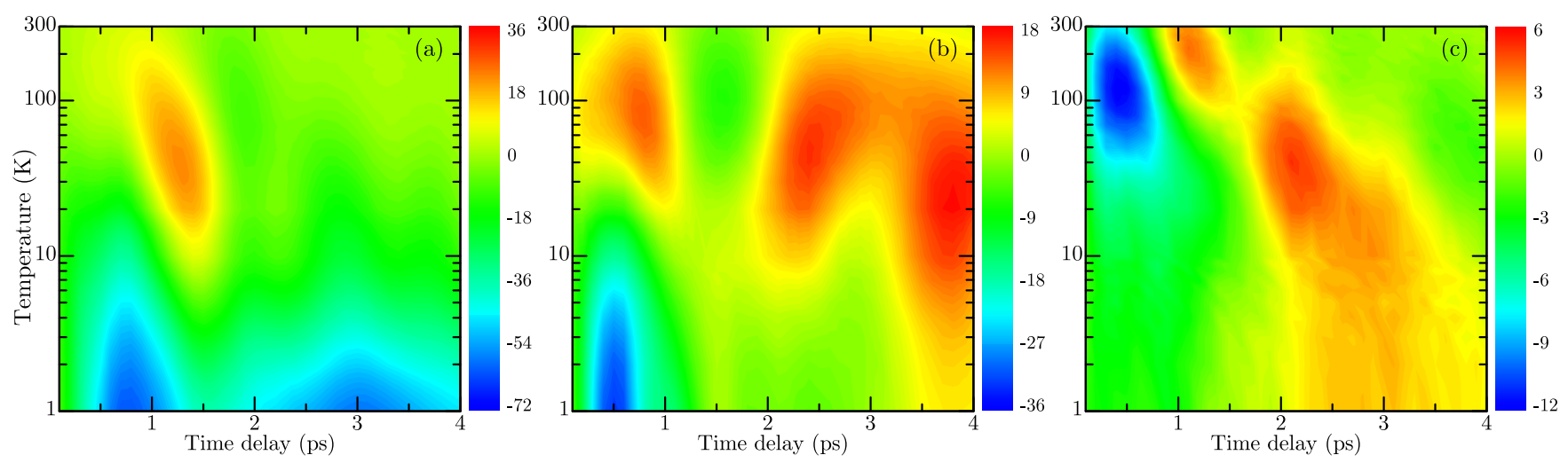

FIG. 4. Classically calculated permanent values of the dipole signals (a) $\left\langle\mu_{X}\right\rangle_{p}$, (b) $\left\langle\mu_{Y}\right\rangle_{p}$, and (c) $\left\langle\mu_{Z}\right\rangle_{p}$ as functions of temperature, $T$, and time delay between the two THz pulses, $\tau$. The color scales are in the units of millidebye $(\mathrm{mD})$.

sign [58]. As a result, the attained asymptotic values of the orientation factors do not change after the initial dephasing of the ensemble which takes about 30 ps; see Fig. 2.

Quantum mechanically, the notion of well-defined trajectories is invalid, which means that the orientation is simply longlived and eventually will change its sign. Since the kinetic energy Hamiltonian [see Eq. (2)] couples rotational states with different $K$ quantum number, the quantum-mechanical asymmetric top does not have permanently oriented eigenstates. Any initially oriented state will eventually oscillate between being oriented and antioriented, an effect known as dynamical tunneling [69]. For a quantum mechanical chiral rotor, one would formally expect no permanent orientation after turning off all external fields. However, as we show here and as was shown in Refs. [28,30], the tunneling timescale may exceed the excitation timescale by orders of magnitude.

It is important to note that the persistent orientation appearing in the directions of each of the pulses (along the $X$ and $Y$ axes) is not chirality dependent. It was recently shown that single $\mathrm{THz}$ pulses applied to symmetric- and asymmetric-top molecules also induce persistent orientation [53]. In contrast, in the present work both the appearance and permanency of the orientation along the propagation direction (along $Z$ axis) depend on the chirality of the molecule. More specifically, these effects rely on the absence of molecular symmetry; i.e., all three molecular dipole moment components must differ from zero, $\mu_{a} \mu_{b} \mu_{c} \neq 0$ (see Appendix B). For comparison, in the case of optical excitation by laser fields with twisted polarization, the orientation relies on the existence of the off-diagonal elements of the polarizability tensor, which is a property of all chiral molecules as well. In that case, the induced dipole has nonzero projections along all three principal axes, even when the laser field is polarized along only one of the molecular principal axes.

The magnitude of the THz-induced orientation is sensitive to the initial temperature and external field parameters $[52,53,70,71]$. Through our classical simulations, we carried out an extensive study of the dependence of the permanent orientation on temperature, $T$, and the time delay between the two cross-polarized pulses, $\tau$ [see Eq. (5)]. The results are summarized in Fig. 4. The permanent values of the dipole projections [denoted by $\left\langle\mu_{X}\right\rangle_{p},\left\langle\mu_{Y}\right\rangle_{p}$, and $\left\langle\mu_{Z}\right\rangle_{p}$ ] were obtained by following the field-free dynamics for a suffi- ciently long time until a steady state is reached (typically $t>$ $100 \mathrm{ps}$ ). Figure 4 shows that for a given rotational temperature, there are one or several disjoint ranges of $\tau$ giving rise to optimal (largest absolute value) orientation. In general, the optimal time delay between the pulses is shorter for the higher temperature. Note, however, that the temperature dependence of the individual projections $\left\langle\mu_{X}\right\rangle_{p},\left\langle\mu_{Y}\right\rangle_{p}$, and $\left\langle\mu_{Z}\right\rangle_{p}$ is nonmonotonic. For example, at a fixed time delay $\tau \approx 0.5 \mathrm{ps}$, $\left|\left\langle\mu_{Z}\right\rangle_{p}\right|$ increases with temperature up to $T \approx 120 \mathrm{~K}$, after which it decreases.

\section{CONCLUSIONS}

We theoretically demonstrated the phenomenon of fieldfree enantioselective orientation of chiral molecules induced by $\mathrm{THz}$ pulses with twisted polarization. The twisted pulse induces orientations of two types. In the directions of the polarizations of the two pulses forming the twisted pulse, the induced orientations of the two enantiomers are of the same sign. In the direction perpendicular to the plane of polarization of both pulses, however, the orientations of the two enantiomers are of opposite signs. The latter effect relies on molecular chirality, namely on the absence of molecular symmetry, such that the molecular dipole has nonzero projections on all three molecular principal axes. The orientation was shown to persist on a nanosecond timescale after the end of the picosecond pulses. We studied the dependence of the persistent orientation values on the temperature and the time delay between the two cross-polarized THz pulses. The orientation factors were found to be quite robust against the detrimental effects of temperature provided that the time delay is adjusted appropriately. The orientation dynamics on timescales beyond nanoseconds requires a more detailed analysis, as in addition to dynamical tunneling other effects such as collisions and fine structure effects [72] may become important and should be assessed in future works. An orientation persisting on the nanosecond timescale may be measured by means of second-order (or higher order) harmonic generation [73], and could be used for deflection by inhomogeneous electric fields [74,75] (for an extensive review, see Ref. [76]). The enantioselective orientation along the propagation direction may be useful for fast and precise analysis of enantiomeric excess and may facilitate enantioselective separation using 
inhomogeneous fields [77]. In the past [25-30], related effects induced by optical pulses with twisted polarization have been reported, and further exploration will examine the combined effect of $\mathrm{THz}$ and optical fields together that could maximize the difference in orientations between the two enantiomers.

\section{ACKNOWLEDGMENTS}

I.A. gratefully acknowledges support by the Israel Science Foundation (Grant No. 746/15). K.A.N. acknowledges support by the U.S. National Science Foundation Grant No. CHE1665383. I.A. acknowledges support as the Patricia Elman Bildner Professorial Chair. This research was made possible in part by the historic generosity of the Harold Perlman Family.

\section{APPENDIX A: QUALITATIVE DEMONSTRATION OF ENANTIOSELECTIVE ORIENTATION}

In this Appendix, we qualitatively demonstrate the enantioselective orientation of chiral molecules induced by $\mathrm{THz}$ pulses with twisted polarization.

Consider two overlapping $\mathrm{THz}$ pulses propagating along the laboratory $Z$ axis and which are polarized along $X$ and $Y$ axes, respectively. The nonzero matrix elements of the interaction potential can be written as [53]

$$
\begin{aligned}
& \left\langle J K M\left|\hat{H}_{\text {int }}(t)\right| J^{\prime}, K-s, M-p\right\rangle \\
& \quad=\mu_{s}^{(1)} E_{-p}^{(1)}(t)\left\langle J K M\left|D_{p, s}^{1}{ }^{*}(R)\right| J^{\prime}, K-s, M-p\right\rangle,
\end{aligned}
$$

where $s=0, \pm 1$ and $p= \pm 1$ (since $\left.E_{0}^{(1)}=0\right)$. Here $\mu_{s}^{(1)}$ and $E_{p}^{(1)}$ are the dipole moment and electric field, respectively, expressed as spherical tensors of rank 1, with components $\mu_{ \pm 1}^{(1)}=\mp\left(\mu_{b} \pm i \mu_{c}\right) / \sqrt{2}, \mu_{0}^{(1)}=\mu_{a}$, and $E_{ \pm 1}^{(1)}=$ $\mp\left(E_{X} \pm i E_{Y}\right) / \sqrt{2}, E_{0}^{(1)}=E_{Z} . J$ is the value of the total angular momentum, while $K$ and $M$ are the values of the projections on the molecule-fixed $a$ axis and the laboratory-fixed $Z$ axis, respectively. $\hat{H}_{\text {int }}(t)=-\hat{\boldsymbol{\mu}} \cdot \mathbf{E}(t)$ is the molecule-field interaction and $D_{p, s}^{1}{ }^{*}(R)$ is the complex conjugate of the Wigner $D$ matrix, where $R$ denotes the set of the three Euler angles, $(\theta, \phi, \chi)$.

The THz pulses induce dynamics of the $Z$ component of the polarization, defined by

$$
P_{Z}(t)=\sum_{s=-1}^{+1} \mu_{s}^{(1)}\left\langle\Psi(t)\left|D_{0, s}^{1 *}(R)\right| \Psi(t)\right\rangle
$$

where the wave function is given by $|\Psi(t)\rangle=\hat{U}(t, 0)|\Psi(0)\rangle$, with $\hat{U}(t, 0)$ being the evolution operator. Since the discussion here is qualitative, for simplicity we assume that initially the rotor is in the ground rotational state $|J K M\rangle=|000\rangle$, such that $|\Psi(t)\rangle=\hat{U}(t, 0)|000\rangle$.

The evolution operator, $\hat{U}(t, 0)$ can be expanded in a Dyson series

$$
\begin{aligned}
\hat{U}(t, 0) & =\sum_{n=0}^{\infty} \frac{(-i)^{n}}{n !} \int_{0}^{t} d t_{1} \int_{0}^{t} d t_{2} \cdots \int_{0}^{t} d t_{n} \hat{\mathcal{T}} \hat{H}_{\mathrm{int}}\left(t_{1}\right) \hat{H}_{\mathrm{int}}\left(t_{2}\right) \cdots \hat{H}_{\mathrm{int}}\left(t_{n}\right) \\
& =\sum_{n=0}^{\infty} \frac{(-i)^{n}}{n !}\left(\prod_{n} \int_{0}^{t} d t_{n}\right) \hat{\mathcal{T}}\left(\prod_{n} \hat{H}_{\mathrm{int}}\left(t_{n}\right)\right),
\end{aligned}
$$

where $\hat{\mathcal{T}}$ is the time-ordering operator. From the properties of Wigner $3-j$ symbols, it follows that the only nonzero matrix elements, $\left\langle J K M\left|D_{0, s}^{1}{ }^{*}(R)\right| J^{\prime}, K-s, M\right\rangle \neq 0$, are those satisfying $|J-1| \leqslant J^{\prime} \leqslant J+1$. Hence, the polarization [Eq. (A2)] can be rewritten as

$$
P_{Z}(t)=\sum_{s=-1}^{+1} \sum_{n, n^{\prime}=0}^{\infty} \mu_{s}^{(1)} \frac{(-1)^{n^{\prime}} i^{n+n^{\prime}}}{n ! n^{\prime} !}\left(\prod_{n} \int_{0}^{t} d t_{n}\right)\left(\prod_{n^{\prime}} \int_{0}^{t} d t_{n^{\prime}}^{\prime}\right) \hat{\mathcal{T}}\left\langle 000\left|\prod_{n} \hat{H}_{\mathrm{int}}\left(t_{n}\right) D_{0, s}^{1}{ }^{*}(R) \prod \hat{H}_{n^{\prime}}\left(t_{n^{\prime}}^{\prime}\right)\right| 000\right\rangle .
$$

The relative sign of $P_{Z}$ between the two enantiomers can be deduced by considering the integrand appearing in the angle brackets in the above equation, which we denote by $V_{s}^{(S / R)}$ for the left-handed $(S)$ and right-handed $(R)$ enantiomers respectively,

$$
\begin{aligned}
V_{s} & \sim \mu_{s}^{(1)}\left\langle 000\left|\prod_{n} \hat{H}_{\mathrm{int}}\left(t_{n}\right) D_{0, s}^{1}{ }^{*}(R) \prod_{n^{\prime}} \hat{H}_{\mathrm{int}}\left(t_{n^{\prime}}^{\prime}\right)\right| 000\right\rangle \\
& =\mu_{s}^{(1)} \sum_{J_{1} K_{1} M_{1}} \sum_{J_{1}^{\prime} K_{1}^{\prime} M_{1}^{\prime}}\left\langle 000\left|\prod_{n} \hat{H}_{\mathrm{int}}\left(t_{n}\right)\right| J_{1} K_{1} M_{1}\right\rangle\left\langle J_{1} K_{1} M_{1}\left|D_{0, s}^{1}{ }^{*}(R)\right| J_{1}^{\prime} K_{1}^{\prime} M_{1}^{\prime}\right\rangle\left\langle J_{1}^{\prime} K_{1}^{\prime} M_{1}^{\prime}\left|\prod_{n^{\prime}} \hat{H}_{\mathrm{int}}\left(t_{n^{\prime}}^{\prime}\right)\right| 000\right\rangle \\
& =\mu_{s}^{(1)} \sum_{J_{1}^{\prime} J_{1} K_{1} M_{1}}\left\langle 000\left|\prod_{n} \hat{H}_{\mathrm{int}}\left(t_{n}\right)\right| J_{1} K_{1} M_{1}\right\rangle\left\langle J_{1} K_{1} M_{1}\left|D_{0, s}^{1}{ }^{*}(R)\right| J_{1}^{\prime}, K_{1}-s, M_{1}\right\rangle\left\langle J_{1}^{\prime}, K_{1}-s, M_{1}\left|\prod_{n^{\prime}} \hat{H}_{\mathrm{int}}\left(t_{n^{\prime}}^{\prime}\right)\right| 000\right\rangle .
\end{aligned}
$$

Note that for the qualitative argument here, the evolution operator in Eq. (A5) is considered as $\hat{U}(t, 0) \sim \prod_{n} \hat{H}_{\text {int }}\left(t_{n}\right)$.
From Eq. (A5), we know that $V_{s}$ differs from zero only if $\left\langle J_{1}+\right.$ $\left.J_{1}^{\prime}, 2 K_{1}-s, 2 M_{1}\left|\prod_{n} \hat{H}_{\text {int }}\left(t_{n}\right) \prod_{n^{\prime}} \hat{H}_{\text {int }}\left(t_{n^{\prime}}^{\prime}\right)\right| 000\right\rangle \neq 0$. Also, 
TABLE II. Molecular properties of methyl chloride $\left(\mathrm{CH}_{3} \mathrm{Cl}\right)$ and sulfur dioxide $\left(\mathrm{SO}_{2}\right)$ molecules: eigenvalues of the moment of inertia tensor (in atomic units) and components of dipole moment (in Debye) in the frame of molecular principal axes of inertia.

\begin{tabular}{lcc}
\hline \hline Molecule & Moments of inertia & Molecular dipole components \\
\hline Methyl chloride & $I_{a}=20910$ & $\mu_{a}=1.986$ \\
& $I_{b}=251506$ & $\mu_{b}=0$ \\
Sulfur dioxide & $I_{c}=251506$ & $\mu_{c}=0$ \\
& $I_{a}=57812$ & $\mu_{a}=0$ \\
& $I_{b}=323141$ & $\mu_{b}=-1.805$ \\
& $I_{c}=380953$ & $\mu_{c}=0$ \\
\hline \hline
\end{tabular}

according to Eq. (A1), the allowed transitions are between the states with $M$ and $M \pm 1$, because $p= \pm 1$. Hence, it follows from Eq. (A5) that the state changing from 0 to $2 M_{1}$ involves an even number of interaction terms $\left(\hat{H}_{\text {int }}\right)$. Similarly, transitions between states with $K$ quantum number equals 0 and $2 K_{1}-s$ involve $2 l+s$ interaction terms with $s=0$ [see Eq. (A1), with $s=0$ ] and $2 L+s$ interaction terms with $s= \pm 1$ [see Eq. (A1), with $s= \pm 1$ ], where $L$ and $l$ are integers. The dipole moments of two enantiomers of the chiral molecule satisfy $\mu_{ \pm 1}^{(S)}=\mu_{ \pm 1}^{(R)}$ and $\mu_{0}^{(S)}=-\mu_{0}^{(R)}$, such that the nonzero polarization components [see Eq. (A5)] satisfy

$$
\begin{aligned}
\frac{V_{s}^{(S)}}{V_{s}^{(R)}} & =\frac{\mu_{s}^{(S)}}{\mu_{s}^{(R)}}\left(\frac{\mu_{-1}^{(S)}}{\mu_{-1}^{(R)}}\right)^{L^{\prime}}\left(\frac{\mu_{0}^{(S)}}{\mu_{0}^{(R)}}\right)^{2 l+s}\left(\frac{\mu_{1}^{(S)}}{\mu_{1}^{(R)}}\right)^{2 L+s-L^{\prime}} \\
& =\frac{\mu_{s}^{(S)}}{\mu_{s}^{(R)}}\left(\frac{\mu_{0}^{(S)}}{\mu_{0}^{(R)}}\right)^{s}=-1,
\end{aligned}
$$

where $s=0, \pm 1$, and $L^{\prime}$ is an integer. As one can see, the components of polarization for the two enantiomers are of opposite signs, and thereby the polarizations satisfy $P_{Z}^{(S)}(t)=$ $-P_{Z}^{(R)}(t)$

\section{APPENDIX B: ORIENTATION OF NONCHIRAL MOLECULES}

In this Appendix, we presents the orientation signals along the three laboratory axes for the methyl chloride $\left(\mathrm{CH}_{3} \mathrm{Cl}\right)$ and sulfur dioxide $\left(\mathrm{SO}_{2}\right)$ molecules excited by a pair of crosspolarized $\mathrm{THz}$ pulses.

Table II summarizes the parameters of methyl chloride and sulfur dioxide. Molecular moments of inertia and the
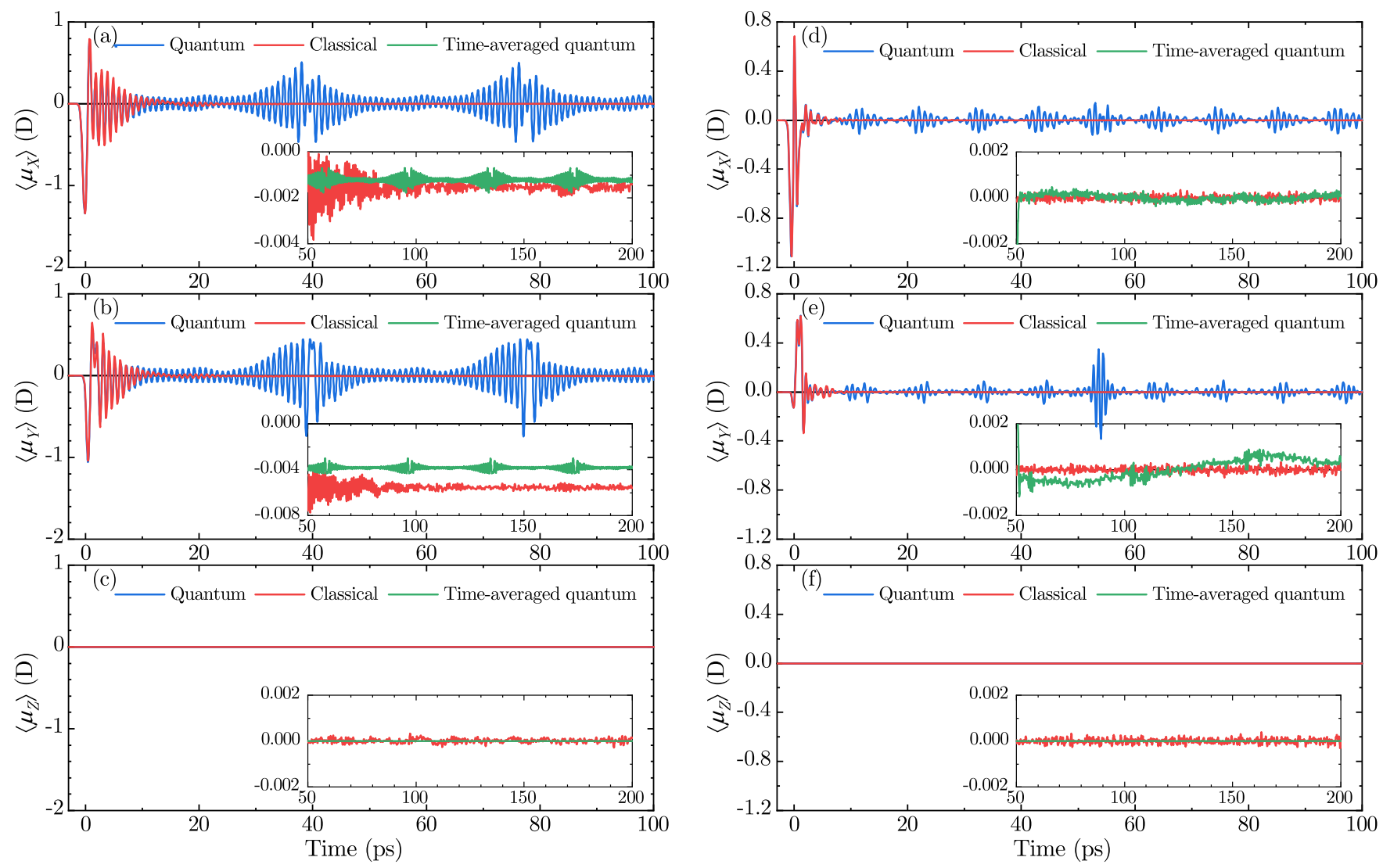

FIG. 5. Thermally averaged $X, Y, Z$ projections of the dipole moment in the laboratory frame, $\left\langle\mu_{X}\right\rangle,\left\langle\mu_{Y}\right\rangle$, and $\left\langle\mu_{Z}\right\rangle$ as functions of time for [(a)-(c)] $\mathrm{CH}_{3} \mathrm{Cl}$ and [(d)-(f)] $\mathrm{SO}_{2}$ molecules. The results of quantum and classical simulations are shown in blue and red lines, respectively. Here, $N=10^{8}$ molecules are used for the classical Monte Carlo simulations. The green curves represent the sliding time average defined by $\overline{\left\langle\mu_{i}\right\rangle(t)}=(\Delta t)^{-1} \int_{t-\Delta t / 2}^{t+\Delta t / 2} d t^{\prime}\left\langle\mu_{i}\right\rangle\left(t^{\prime}\right)$, where [(a)-(c)] $\Delta t=38.2 \mathrm{ps}$ and [(d)-(f)] $\Delta t=100 \mathrm{ps}$, respectively. The insets show the magnified portions of the signals. 
components of molecular dipole moments were computed with the help of GAUSSIAN software package (method: CAMB3LYP/aug-cc-pVTZ) [62].

Figure 5 shows the orientation signals along the three laboratory axes, $\left\langle\mu_{X}\right\rangle(t),\left\langle\mu_{Y}\right\rangle(t)$, and $\left\langle\mu_{Z}\right\rangle(t)$, for two nonchiral molecules. The first one is the symmetric-top methyl chloride $\left(\mathrm{CH}_{3} \mathrm{Cl}\right)$, in which the molecular dipole moment is along the symmetry axis, $a$ axis $\left(\mu_{b}=\mu_{c}=0\right.$; see Table II). The second molecule, sulfur dioxide $\left(\mathrm{SO}_{2}\right)$, is an asymmetric-top molecule in which the molecular dipole moment is along the molecular $b$ axis $\left(\mu_{a}=\mu_{c}=0\right.$; see Table II). Here the initial temperature is $T=5 \mathrm{~K}$. The electric field of the THz pulses is modeled by $\mathbf{E}(t)=E_{0}\left[f(t) \mathbf{e}_{X}+f(t-\tau) \mathbf{e}_{Y}\right]$, where $E_{0}=8.0 \mathrm{MV} / \mathrm{cm}$ is the peak amplitude, the time dependence is defined by $f(t)=\left(1-2 a t^{2}\right) e^{-a t^{2}}$ with $a=$ $3.06 \mathrm{ps}^{-2}, \tau=0.80 \mathrm{ps}$ is the time delay between the maxima of the two pulses, and $\mathbf{e}_{X}$ and $\mathbf{e}_{Y}$ are the unit vectors along the laboratory $X$ and $Y$ axes, respectively. The quantum and classical results are in good agreement. As expected, both the symmetric-top [Figs. 5(a) and 5(b)] and nonchiral asymmetric-top [Figs. 5(d) and 5(e)] molecules immediately respond to the $X$-polarized and $Y$-polarized pulses. However, in contrast to chiral molecules (see Fig. 2 in the main text), the $Z$ projection of the dipole moment remains zero [see Figs. 5(c) and 5(f)]. Note that $\left\langle\mu_{Z}\right\rangle$ calculated quantum mechanically is identically zero, while the small-amplitude oscillations appearing in the classical results are due to the finite number of molecules in the ensemble. The amplitude of the oscillations is expected to scale as $1 / \sqrt{N}$, where $N$ is the number of molecules used in the Monte Carlo simulations. The lack of orientation in the cases of symmetric-top or nonchiral asymmetric-top molecules indicates that all three molecular dipole components $\left(\mu_{a}, \mu_{b}, \mu_{c}\right)$ are indeed required for inducing the perpendicular (in the $Z$ direction) orientation. Other combinations were considered numerically as well (not shown), e.g., asymmetric-top molecule having two nonzero molecular dipole components. In all cases, there is no perpendicular orientation, i.e., $\left\langle\mu_{Z}\right\rangle=0$.
[1] L. Pasteur, Sur les relations qui peuvent exister entre la forme crystalline, la composition chimique et le sens de la polarization rotatoire, Ann. Phys. Chem. 24, 442 (1848).

[2] S. F. Mason, The foundations of classical stereochemistry, in Topics in Stereochemistry (John Wiley \& Sons, New York, 2007), pp. 1-34.

[3] F. A. Cotton, Chemical Applications of Group Theory, 3rd ed. (John Wiley \& Sons, New York, 1990).

[4] R. Berger and J. Stohner, Parity violation, Wiley Interdiscip. Rev. Comput. Mol. Sci 9, e1396 (2019).

[5] E. Hirota, Triple resonance for a three-level system of a chiral molecule, Proc. Jpn. Acad., Ser. B 88, 120 (2012).

[6] D. Patterson, M. Schnell, and J. M. Doyle, Enantiomer-specific detection of chiral molecules via microwave spectroscopy, Nature (London) 497, 475 (2013).

[7] D. Patterson and J. M. Doyle, Sensitive Chiral Analysis via Microwave Three-Wave Mixing, Phys. Rev. Lett. 111, 023008 (2013).

[8] D. Patterson and M. Schnell, New studies on molecular chirality in the gas phase: Enantiomer differentiation and determination of enantiomeric excess, Phys. Chem. Chem. Phys. 16, 11114 (2014).

[9] V. A. Shubert, D. Schmitz, and M. Schnell, Enantiomersensitive spectroscopy and mixture analysis of chiral molecules containing two stereogenic centers-microwave three-wave mixing of menthone, J. Mol. Spectrosc. 300, 31 (2014).

[10] K. K. Lehmann, Theory of enantiomer-specific microwave spectroscopy, in Frontiers and Advances in Molecular Spectroscopy (Elsevier, Amsterdam, 2018), pp. 713-743.

[11] M. Leibscher, T. F. Giesen, and C. P. Koch, Principles of enantio-selective excitation in three-wave mixing spectroscopy of chiral molecules, J. Chem. Phys. 151, 014302 (2019).

[12] B. Ritchie, Theory of the angular distribution of photoelectrons ejected from optically active molecules and molecular negative ions, Phys. Rev. A 13, 1411 (1976).
[13] N. Böwering, T. Lischke, B. Schmidtke, N. Müller, T. Khalil, and U. Heinzmann, Asymmetry in Photoelectron Emission from Chiral Molecules Induced by Circularly Polarized Light, Phys. Rev. Lett. 86, 1187 (2001).

[14] C. Lux, M. Wollenhaupt, T. Bolze, Q. Liang, J. Köhler, C. Sarpe, and T. Baumert, Circular dichroism in the photoelectron angular distributions of camphor and fenchone from multiphoton ionization with femtosecond laser pulses, Angew. Chem. Int. Ed. Eng. 51, 5001 (2012).

[15] S. Beaulieu, A. Comby, A. Clergerie, J. Caillat, D. Descamps, N. Dudovich, B. Fabre, R. Géneaux, F. Légaré, S. Petit, B. Pons, G. Porat, T. Ruchon, R. Taïeb, V. Blanchet, and Y. Mairesse, Attosecond-resolved photoionization of chiral molecules, Science 358, 1288 (2017).

[16] S. Beaulieu, A. Comby, D. Descamps, B. Fabre, G. A. Garcia, R. Géneaux, A. G. Harvey, F. Légaré, Z. Mašín, L. Nahon, A. F. Ordonez, S. Petit, B. Pons, Y. Mairesse, O. Smirnova, and V. Blanchet, Photoexcitation circular dichroism in chiral molecules, Nat. Phys. 14, 484 (2018).

[17] K. Banerjee-Ghosh, O. Ben Dor, F. Tassinari, E. Capua, S. Yochelis, A. Capua, S.-H. Yang, S. S. P. Parkin, S. Sarkar, L. Kronik, L. T. Baczewski, R. Naaman, and Y. Paltiel, Separation of enantiomers by their enantiospecific interaction with achiral magnetic substrates, Science 360, 1331 (2018).

[18] M. Pitzer, M. Kunitski, A. S. Johnson, T. Jahnke, H. Sann, F. Sturm, L. Ph. H. Schmidt, H. Schmidt-Böcking, R. Dörner, J. Stohner, J. Kiedrowski, M. Reggelin, S. Marquardt, A. Schießer, R. Berger, and M. S. Schöffler, Direct determination of absolute molecular stereochemistry in gas phase by Coulomb explosion imaging, Science 341, 1096 (2013).

[19] P. Herwig, K. Zawatzky, M. Grieser, O. Heber, B. JordonThaden, C. Krantz, O. Novotný, R. Repnow, V. Schurig, D. Schwalm, Z. Vager, A. Wolf, O. Trapp, and H. Kreckel, Imaging the absolute configuration of a chiral epoxide in the gas phase, Science 342, 1084 (2013). 
[20] K. Fehre, S. Eckart, M. Kunitski, M. Pitzer, S. Zeller, C. Janke, D. Trabert, J. Rist, M. Weller, A. Hartung, L. Ph. H. Schmidt, T. Jahnke, R. Berger, R. Dörner, and M. S. Schöffler, Enantioselective fragmentation of an achiral molecule in a strong laser field, Sci. Adv. 5, eaau7923 (2019).

[21] R. Cireasa, A. E. Boguslavskiy, B. Pons, M. C. H. Wong, D. Descamps, S. Petit, H. Ruf, N. Thiré, A. Ferré, J. Suarez, J. Higuet, B. E. Schmidt, A. F. Alharbi, F. Légaré, V. Blanchet, B. Fabre, S. Patchkovskii, O. Smirnova, Y. Mairesse, and V. R. Bhardwaj, Probing molecular chirality on a sub-femtosecond timescale, Nat. Phys. 11, 654 (2015).

[22] M. Shapiro, E. Frishman, and P. Brumer, Coherently Controlled Asymmetric Synthesis with Achiral Light, Phys. Rev. Lett. 84, 1669 (2000).

[23] Y. Inoue and V. Ramamurthy, eds., Chiral Photochemistry, 1st ed. (CRC Press, Boca Raton, FL, 2004).

[24] S. Eibenberger, J. Doyle, and D. Patterson, Enantiomer-Specific State Transfer of Chiral Molecules, Phys. Rev. Lett. 118, 123002 (2017).

[25] A. Yachmenev and S. N. Yurchenko, Detecting Chirality in Molecules by Linearly Polarized Laser Fields, Phys. Rev. Lett. 117, 033001 (2016).

[26] E. Gershnabel and I. Sh. Averbukh, Orienting Asymmetric Molecules by Laser Fields with Twisted Polarization, Phys. Rev. Lett. 120, 083204 (2018).

[27] I. Tutunnikov, E. Gershnabel, S. Gold, and I. Sh. Averbukh, Selective orientation of chiral molecules by laser fields with twisted polarization, J. Phys. Chem. Lett. 9, 1105 (2018).

[28] I. Tutunnikov, J. Floß, E. Gershnabel, P. Brumer, and I. Sh. Averbukh, Laser-induced persistent orientation of chiral molecules, Phys. Rev. A 100, 043406 (2019).

[29] A. A. Milner, J. A. M. Fordyce, I. MacPhail-Bartley, W. Wasserman, V. Milner, I. Tutunnikov, and I. Sh. Averbukh, Controlled Enantioselective Orientation of Chiral Molecules with an Optical Centrifuge, Phys. Rev. Lett. 122, 223201 (2019).

[30] I. Tutunnikov, J. Floß, E. Gershnabel, P. Brumer, I. Sh. Averbukh, A. A. Milner, and V. Milner, Observation of persistent orientation of chiral molecules by a laser field with twisted polarization, Phys. Rev. A 101, 021403(R) (2020).

[31] S. Fleischer, Y. Khodorkovsky, Y. Prior, and I. Sh. Averbukh, Controlling the sense of molecular rotation, New J. Phys. 11, 105039 (2009).

[32] K. Kitano, H. Hasegawa, and Y. Ohshima, Ultrafast Angular Momentum Orientation by Linearly Polarized Laser Fields, Phys. Rev. Lett. 103, 223002 (2009).

[33] Y. Khodorkovsky, K. Kitano, H. Hasegawa, Y. Ohshima, and I. Sh. Averbukh, Controlling the sense of molecular rotation: Classical versus quantum analysis, Phys. Rev. A 83, 023423 (2011).

[34] S. Zhdanovich, A. A. Milner, C. Bloomquist, J. Floß, I. Sh. Averbukh, J. W. Hepburn, and V. Milner, Control of Molecular Rotation with a Chiral Train of Ultrashort Pulses, Phys. Rev. Lett. 107, 243004 (2011).

[35] J. Floß and I. Sh. Averbukh, Molecular spinning by a chiral train of short laser pulses, Phys. Rev. A 86, 063414 (2012).

[36] Y. Kida, S.-i. Zaitsu, and T. Imasaka, Stimulated rotational Raman scattering by a polarization-modulated femtosecond pulse, Phys. Rev. A 77, 063802 (2008).

[37] Y. Kida, S.-i. Zaitsu, and T. Imasaka, Coherent molecular rotations induced by a femtosecond pulse consisting of two orthogonally polarized pulses, Phys. Rev. A 80, 021805(R) (2009).

[38] G. Karras, M. Ndong, E. Hertz, D. Sugny, F. Billard, B. Lavorel, and O. Faucher, Polarization Shaping for Unidirectional Rotational Motion of Molecules, Phys. Rev. Lett. 114, 103001 (2015).

[39] E. Prost, H. Zhang, E. Hertz, F. Billard, B. Lavorel, P. Bejot, J. Zyss, I. Sh. Averbukh, and O. Faucher, Third-order-harmonic generation in coherently spinning molecules, Phys. Rev. A 96, 043418 (2017).

[40] K. Mizuse, N. Sakamoto, R. Fujimoto, and Y. Ohshima, Direct imaging of direction-controlled molecular rotational wave packets created by a polarization-skewed double-pulse, Phys. Chem. Chem. Phys. 22, 10853 (2020).

[41] J. Karczmarek, J. Wright, P. Corkum, and M. Ivanov, Optical Centrifuge for Molecules, Phys. Rev. Lett. 82, 3420 (1999).

[42] D. M. Villeneuve, S. A. Aseyev, P. Dietrich, M. Spanner, M. Yu. Ivanov, and P. B. Corkum, Forced Molecular Rotation in an Optical Centrifuge, Phys. Rev. Lett. 85, 542 (2000).

[43] L. Yuan, S. W. Teitelbaum, A. Robinson, and A. S. Mullin, Dynamics of molecules in extreme rotational states, Proc. Natl. Acad. Sci. U.S.A. 108, 6872 (2011).

[44] A. Korobenko, A. A. Milner, and V. Milner, Direct Observation, Study, and Control of Molecular Superrotors, Phys. Rev. Lett. 112, 113004 (2014).

[45] A. Korobenko, Control of molecular rotation with an optical centrifuge, J. Phys. B 51, 203001 (2018).

[46] S. Fleischer, Y. Zhou, R. W. Field, and K. A. Nelson, Molecular Orientation and Alignment by Intense Single-Cycle THz Pulses, Phys. Rev. Lett. 107, 163603 (2011).

[47] K. Kitano, N. Ishii, N. Kanda, Y. Matsumoto, T. Kanai, M. Kuwata-Gonokami, and J. Itatani, Orientation of jet-cooled polar molecules with an intense single-cycle $\mathrm{THz}$ pulse, Phys. Rev. A 88, 061405(R) (2013).

[48] K. N. Egodapitiya, S. Li, and R. R. Jones, Terahertz-Induced Field-Free Orientation of Rotationally Excited Molecules, Phys. Rev. Lett. 112, 103002 (2014).

[49] P. Babilotte, K. Hamraoui, F. Billard, E. Hertz, B. Lavorel, O. Faucher, and D. Sugny, Observation of the field-free orientation of a symmetric-top molecule by terahertz laser pulses at high temperature, Phys. Rev. A 94, 043403 (2016).

[50] L. H. Coudert, Optimal orientation of an asymmetric top molecule with terahertz pulses, J. Chem. Phys. 146, 024303 (2017).

[51] R. Damari, D. Rosenberg, and S. Fleischer, Coherent Radiative decay of Molecular Rotations: A Comparative Study of Terahertz-Oriented Versus Optically Aligned Molecular Ensembles, Phys. Rev. Lett. 119, 033002 (2017).

[52] R. Tehini, K. Hamraoui, and D. Sugny, Shaping of the time evolution of field-free molecular orientation by $\mathrm{THz}$ laser pulses, Phys. Rev. A 99, 033419 (2019).

[53] L. Xu, I. Tutunnikov, E. Gershnabel, Y. Prior, and I. Sh Averbukh, Long-Lasting Molecular Orientation Induced by a Single Terahertz Pulse, Phys. Rev. Lett. 125, 013201 (2020).

[54] R. V. Krems, Molecules in Electromagnetic Fields: From Ultracold Physics to Controlled Chemistry (Wiley, New York, 2018).

[55] C. P. Koch, M. Lemeshko, and D. Sugny, Quantum control of molecular rotation, Rev. Mod. Phys. 91, 035005 (2019).

[56] R. N. Zare, Angular Momentum: Understanding Spatial Aspects in Chemistry and Physics (Wiley, New York, 1988). 
[57] R. B. Sidje, EXPOKIT: A software package for computing matrix exponentials, ACM Trans. Math. Softw. 24, 130 (1998).

[58] H. Goldstein, C. Poole, and J. Safko, Classical Mechanics (Addison Wesley, San Francisco, CA, 2002).

[59] E. A. Coutsias and L. Romero, The quaternions with an application to rigid body dynamics, Sandia Technical Report, SAND2004-0153, 2004 (unpublished).

[60] J. B. Kuipers, Quaternions and Rotation Sequences: A Primer with Applications to Orbits, Aerospace, and Virtual Reality (Princeton University Press, Princeton, NJ, 1999).

[61] S. M. LaValle, Planning Algorithms (Cambridge University Press, New York, 2006).

[62] M. J. Frisch, G. W. Trucks, H. B. Schlegel, G. E. Scuseria, M. A. Robb, J. R. Cheeseman, G. Scalmani, V. Barone, G. A. Petersson, H. Nakatsuji, X. Li, M. Caricato, A. V. Marenich, J. Bloino, B. G. Janesko, R. Gomperts, B. Mennucci, H. P. Hratchian, J. V. Ortiz, A. F. Izmaylov et al., Gaussian 16 Revision A. 03 (Gaussian Inc., Wallingford, CT, 2016).

[63] M. Clerici, M. Peccianti, B. E. Schmidt, L. Caspani, M. Shalaby, M. Giguère, A. Lotti, A. Couairon, F. Légaré, T. Ozaki, D. Faccio, and R. Morandotti, Wavelength Scaling of Terahertz Generation by Gas Ionization, Phys. Rev. Lett. 110, 253901 (2013).

[64] T. I. Oh, Y. J. Yoo, Y. S. You, and K. Y. Kim, Generation of strong terahertz fields exceeding $8 \mathrm{MV} / \mathrm{cm}$ at $1 \mathrm{kHz}$ and realtime beam profiling, Appl. Phys. Lett. 105, 041103 (2014).

[65] D. Kuk, Y. J. Yoo, E. W. Rosenthal, N. Jhajj, H. M. Milchberg, and K. Y. Kim, Generation of scalable terahertz radiation from cylindrically focused two-color laser pulses in air, Appl. Phys. Lett. 108, 121106 (2016).

[66] I. Sh. Averbukh and N. F. Perelman, Fractional revivals: Universality in the long-term evolution of quantum wave packets beyond the correspondence principle dynamics, Phys. Lett. A 139, 449 (1989).
[67] P. M. Felker, Rotational coherence spectroscopy: Studies of the geometries of large gas-phase species by picosecond timedomain methods, J. Phys. Chem. 96, 7844 (1992).

[68] R. W. Robinett, Quantum wave packet revivals, Phys. Rep 392, 1 (2004).

[69] S. Keshavamurthy and P. Schlagheck, Dynamical Tunneling: Theory and Experiment (CRC Press, Boca Raton, FL, 2011).

[70] M. Lapert and D. Sugny, Field-free molecular orientation by terahertz laser pulses at high temperature, Phys. Rev. A 85 , 063418 (2012).

[71] C.-C. Shu and N. E. Henriksen, Field-free molecular orientation induced by single-cycle $\mathrm{THz}$ pulses: The role of resonance and quantum interference, Phys. Rev. A 87, 013408 (2013).

[72] E. F. Thomas, A. A. Søndergaard, B. Shepperson, N. E. Henriksen, and H. Stapelfeldt, Hyperfine-Structure-Induced Depolarization of Impulsively Aligned $\mathrm{I}_{2}$ Molecules, Phys. Rev. Lett. 120, 163202 (2018).

[73] E. Frumker, C. T. Hebeisen, N. Kajumba, J. B. Bertrand, H. J. Wörner, M. Spanner, D. M. Villeneuve, A. Naumov, and P. B. Corkum, Oriented Rotational Wave-Packet Dynamics Studies via High Harmonic Generation, Phys. Rev. Lett. 109, 113901 (2012).

[74] E. Gershnabel and I. Sh. Averbukh, Electric deflection of rotating molecules, J. Chem. Phys. 134, 054304 (2011).

[75] E. Gershnabel and I. Sh. Averbukh, Deflection of rotating symmetric top molecules by inhomogeneous fields, J. Chem. Phys. 135, 084307 (2011).

[76] Y.-P. Chang, D. A. Horke, S. Trippel, and J. Küpper, Spatiallycontrolled complex molecules and their applications, Int. Rev. Phys. Chem. 34, 557 (2015).

[77] A. Yachmenev, J. Onvlee, E. Zak, A. Owens, and J. Küpper, Field-Induced Diastereomers for Chiral Separation, Phys. Rev. Lett. 123, 243202 (2019). 